\title{
Influence of reaming intramedullary nailing on MSC population after surgical treatment of patients with long bone fracture
}

\author{
IOANNIS SPERELAKIS ${ }^{1}$, ELIZA TSITOURA ${ }^{2}$, CHARA KOUTOULAKI ${ }^{2}$, \\ SEMELI MASTRODIMOU ${ }^{2}$, THEODOROS H. TOSOUNIDIS ${ }^{1}$, DEMETRIOS A. SPANDIDOS ${ }^{3}$, \\ KATERINA M. ANTONIOU ${ }^{2}$ and GEORGE KONTAKIS ${ }^{1}$ \\ ${ }^{1}$ Department of Orthopedics and Traumatology, University of Crete School of Medicine; \\ ${ }^{2}$ Department of Respiratory Medicine, University General Hospital of Heraklion, \\ Laboratory of Molecular and Cellular Pneumonology, Medical School, University of Crete; \\ ${ }^{3}$ Laboratory of Clinical Virology, Medical School, University of Crete, 71003 Heraklion, Greece
}

Received June 8, 2020; Accepted July 9, 2020

DOI: $10.3892 / \mathrm{mmr} .2020 .11320$

\begin{abstract}
Reamed intramedullary nailing (RIN) is a surgical method of choice for treatment of diaphyseal fractures. This procedure affects the biological environment of bone tissue locally and systemically. This study investigated the influence of RIN on mesenchymal stem cells (MSCs) in patients with long bone fractures. The axis of C-X-C motif chemokine receptor 4 (CXCR4)/stromal cell-derived factor 1 (SDF-1) was selected since it is considered as major pathway for MSC homing and migration. Iliac crest bone marrow (IC-BM) samples and blood samples were collected at two different time points. One sample was collected before the RIN (BN) and the other immediately after RIN (AN). BM-MSCs were cultured and RT-qPCR was performed for CXCR4 mRNA levels and ELISA for the SDF-1 sera levels. The experimental study revealed that there was a correlation between the increase of SDF-1 levels in peripheral blood and a decrease in the levels of CXCR4 in MSCs in the IC-BM following RIN. The levels of SDF-1 showed a significant increase in the sera of patients after RIN. In conclusion, the present study is the first providing evidence of the effects of RIN on MSC population via the CXCR4/SDF-1 axis. The levels of serum SDF-1 factor were elevated after RIN while increased levels of SDF-1 in peripheral blood were inversely correlated with the mRNA levels of CXCR4 on BM-MSCs after RIN. Therefore, this
\end{abstract}

Correspondence to: Dr Katerina M. Antoniou, Department of Respiratory Medicine, University General Hospital of Heraklion, Laboratory of Molecular and Cellular Pneumonology, Medical School, University of Crete, 71003 Heraklion, Greece

E-mail: kantoniou@med.uoc.gr

Key words: reaming intramedullary nailing, long bone fracture, mesenchymal stem cells, SDF-1, CXCR4 study contributes to enlighten the systematic effects of RIN on the population of MSCs at a cellular level.

\section{Introduction}

Intramedullary nailing (IN) is a method of choice for treatment of long bone fractures. This treatment has two choices of bone preparation for fixation: un-reamed and reamed technique. The reamed IN (RIN) method uses a long reamer instrument in order to open the fractured bone medullar canal to insert the nail in a more fitted way to the fractured long bone (1). The nailing procedure affects the biological environment of the bone and thus the fracture healing (2).

During RIN procedure several changes take place in the medullary canal. The first parameter that is affected is the intramedullary pressure which rises as a result of the effect of the reamer device in the bone canal. The normal blood flow in the long bones is centripetal. Under the increase of the intramedullary pressure, the blood flow is reversed to centrifugal $(3,4)$. The reversed flow drifts medullary content into the systematic circulation through the metaphyseal vascular systems (5). The medullary content is called 'debris' and several investigations were performed in order to reveal its components. It was shown that 'debris' is a source of mesenchymal stem cells (MSCs) (6), which can be cultured in vitro. RIN releases several growth factors which along with the cell debris promote an osteogenic effect. MSCs are multipotent stem cells which can differentiate into several tissues as adipocytes, chondrocytes and osteocytes. Their ability to regenerate human body tissues directed a large amount of research to investigate their possible role in several diseases and most importantly resulted in their usage as treatment in the field of medical practice of regenerative medicine (7). Mobilization and homing of MSCs from the bone marrow (BM) pool occurs following tissue damage such as burn trauma, muscle damage and cardiac stroke (8-12). Several molecules are implicated in the MSC homing and migration. Among the chemokines tested in an in vitro migration model, stromal cell-derived factor 1 (SDF-1) 
showed significant chemotactic influence. BM-MSCs express C-X-C motif chemokine receptor 4 (CXCR4) which circulates toward SDF-1 gradients (13). Under normal circumstances $\mathrm{BM}-\mathrm{MSCs}$ remain at the $\mathrm{BM}$ niche under the action of SDF-1/CXCR4 axis. The CXCR4/SDF-1 axis is considered to be the key effector of MSC homing and migration. In case of inflammation following tissue damage, such as RIN, several enzymes released following the release of chemokines affect the integrity of SDF-1 and CXCR4 and promote mobilization by breaking the 'anchors' of MSCs inside the BM pool $(14,15)$. There are data suggesting that platelet-derived growth factors $\mathrm{AA}$ and $\mathrm{BB}$ can influence MSC migration at remote sites from the initial injury in patients with fractures by changing the cell surface receptors CD140a and CD140b, which lead to MSC proliferation (16). However, there are to date no studies addressing the effect of CXCR4/SDF-1 axis in patients with fractures. The purpose of the present study was to evaluate the effects of RIN on the MSC pool in the iliac crest BM (IC-BM) in patients treated for long bone fractures and the possible role of CXCR4/SDF-1 axis.

We report increased serum levels of SDF-1 following RIN, inversely correlated to CXCR4 mRNA level alterations in IC-BM MSCs. These results indicate evidence for a systemic SDF-1 up-regulation and an influence of RIN at MSCs pool, a remote site from RIN.

\section{Patients and methods}

Selection of patients. In total, 15 patients were included. They were diagnosed with fracture on the diaphysis of a long bone, femur or tibia. The selected therapy was surgical and specifically IN of the injured bone. The IN procedure included reaming (RIN) of the bone canal in all cases. The inclusion criteria were: i) age $\leq 65$ years; ii) no co-morbidities; iii) no history of steroid medication; and iv) the surgery to take place within the first $24 \mathrm{~h}$ following the incident of fracture. The Ethics Committee of the University Hospital of Heraklion approved the experimental study protocol (Decision no. 185, Protocol no. 14880/14/1/2015). All patients included in the study were informed and written consent was obtained.

BM-MSCs isolation and culture. BM samples were aspirated from the anterior posterior iliac crest and cultured in vitro according to a previously described protocol $(17,18)$. For each patient two BM samples of $10 \mathrm{ml}$ each were aspirated, one before the surgical procedure and a second immediately after the end of the surgery. Briefly, BM mononuclear cells (BM-MNCs) isolated by Histopaque-1077 (Sigma-Aldrich; Merck KGaA) centrifugation, were cultured in Dulbecco's modified Eagle's medium-low glucose (DMEM-LG; Gibco; Thermo Fisher Scientific, Inc.) in the presence of $10 \%$ fetal calf serum (FCS; HyClone; GE Healthcare Life Sciences) and $100 \mathrm{IU} / \mathrm{ml}$ penicillin-streptomycin (PS; Gibco; Thermo Fisher Scientific, Inc.) consisting the MSC medium). Cells were cultured at a concentration of $2 \times 10^{5}$ cells $/ \mathrm{cm}^{2}$ in $25 \mathrm{~cm}^{2}$ flasks at $37^{\circ} \mathrm{C}$ temperature $/ 5 \% \mathrm{CO}_{2}$ humidified atmosphere. One to three days after seeding, floating cells were removed and the medium was replaced by fresh MSC medium. Cells were trypsinized $(\mathrm{P})$ when the confluence reached $70-90 \%$ by $0.25 \%$ trypsin-1 mM EDTA (Gibco; Thermo Fisher Scientific,
Inc.). Cell counts in passages $\mathrm{P} 1$ and $\mathrm{P} 2$ were performed and doubling time (DT) was calculated according to the formula:

$\mathrm{DT}=t / n=t \times \log (2) / \log ($ cells harvested/cells inoculated),

where $t$ is the time between initial plating and harvest for the respective passage.

MSCs were characterized by their morphologic (all displayed the characteristic spindle-shape) and immunophenotypic features (data not shown) (19).

Blood sample collection. Peripheral blood samples were obtained from all participants at the same two time points as the BM samples. The first was obtained before the surgery and the second immediately after the end of the surgical procedure. All samples were processed appropriately according to standard procedures (7) and serum was stored at $-80^{\circ} \mathrm{C}$.

Reverse transcription-quantitative polymerase chain reaction assay. One million cells from all $\mathrm{P} 2 \mathrm{BM}-\mathrm{MSCs}$ samples were homogenized in TRIzol reagent (Molecular Research Center Inc.). Total RNA from both time point samples was extracted, and cDNA was synthesized by RT with the Thermo Scientific RevertAid ${ }^{\mathrm{TM}}$ First Strand cDNA Synthesis kit (Thermo Fisher Scientific, Inc.). The mRNA expression was measured using a RT-qPCR assay with SYBR-Green I. Glyceraldehyde-3-phosphate dehydrogenase (GAPDH) was used as the internal control, in order to normalize SDF-1 and CXCR4 expression levels. Relative expression levels of the examined factors per sample were calculated according to $2^{-\Delta \Delta \mathrm{Ct}}$ method (20) followed by $\log 2$ transformation: $\Delta \Delta \mathrm{CT}=\{(\mathrm{Ct} \mathrm{CXCR} 4-\mathrm{Ct} \mathrm{GAPDH})$ sample after $\}-\{(\mathrm{Ct}$ CXCR4-Ct GAPDH) sample before $\}$.

The mRNA-specific primers used are listed in Table I.

Serum SDF-1. SDF-1 levels in sera were evaluated by ELISA method (Quantikine; R\&D Systems).

Statistical analysis. The results are expressed as mean \pm SD unless otherwise stated. Pearson's rank coefficient (rho) was used to evaluate correlations of the expression of the molecules CXCR4 and SDF1. Wilcoxon matched-pairs signed rank test was used to compare DT from the two time point collections. Paired t-test was used to compare serum SDF-1 levels before and after nailing. Analysis was performed using GraphPad Prism v.6 statistical program (GraphPad Software, Inc). $\mathrm{P}<0.05$ was considered to indicate a statistically significant difference.

\section{Results}

Demographics and patient data. A total of 15 patients were included to the experimental study. All the patients were diagnosed with fracture at the diaphysis of the femur or the tibia. The average age of patients was $34.64 \pm 14.30$ years. The maximum age was 62 years according to the selection criteria and the minimum was 18 years. The ratio between male/female was $10 / 5$. The most common injury occurred in the tibia ( 9 out of 15) and the most common injured lower limb was the right side (8 out of 15). 
Table I. Primer sequences used for RT-qPCR.

Gene

Primer sequence $\left(5^{\prime}-3^{\prime}\right)$

Annealing temperature, ${ }^{\circ} \mathrm{C}$

CXCR4

F: GGTGGTCTATGTTGGCGTCT

55

GAPDH

R: TGGAGTGTGACAGCTTGGAG

F: AGCCACATCGCTCAGACA

53

R: CCAATACGACCAAATCCGTT

RT-qPCR, reverse transcription-quantitative polymerase chain reaction; F, forward; R, reverse.

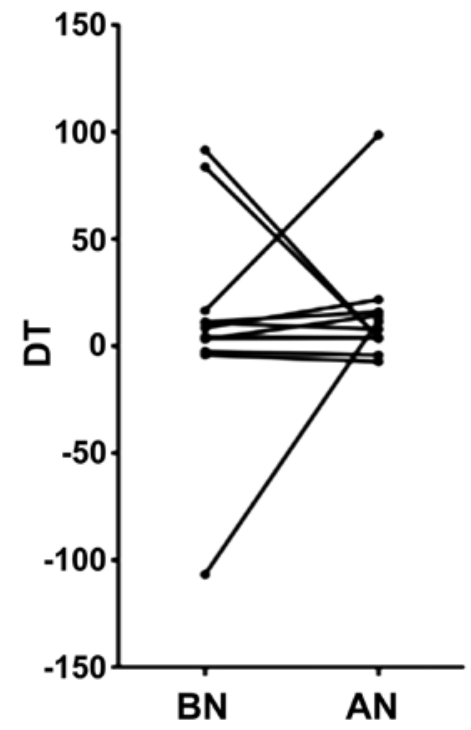

Figure 1. DT of BN and AN samples are similar. DT of MSCs prior to and following RIN procedure according to each patient, calculated as described in methods. DT, doubling time; RIN, reamed intramedullary nailing; BN, before RIN; AN, after RIN.

MSC characteristics and data. IC-BM samples and blood samples were collected at two time points. One sample was collected before the RIN (BN) and the other immediately after RIN (AN). Initially, a possible effect of RIN on cell DT was evaluated. DT was measured for both time point samples of MSCs for 13 out of 15 patients (median \pm SEM: $\mathrm{BN}=4.37 \pm 12.97$ and $\mathrm{AN}=7.87 \pm 6.34$ ). There was no statistical difference between the DT of BN and AN samples (Wilcoxon matched-pairs signed rank test $\Pi=0.54$ ) (Fig. 1).

SDF-1 levels following RIN. SDF-1 levels were compared in the serum between the two time point samples (mean \pm SD: $\mathrm{BN}=1,300 \pm 542.8$ and $\mathrm{AN}=1,633 \pm 632.4$ ) (Fig. 2A). The levels of SDF-1 showed a significant increase in the sera of patients after nailing (paired t-test $\mathrm{p}=0.049)$ (Fig. 2B). The change of the levels of SDF-1 factor was also calculated with 9 out of 12 patients showing an increase in SDF-1 levels. This suggested that nailing influences the levels of SDF-1 factor. There was no statistically significant correlation between age and changes in SDF-1 serum levels (Pearson's $r=-0.27$, $\mathrm{P}=0.4$ ), neither, any correlation between changes in SDF-1 serum levels with the patient's gender (Spearman's $r=-0.19$, $\mathrm{P}=0.6)$ or the DT (BN Spearman's $\mathrm{r}=0.56, \mathrm{P}=0.1, \mathrm{AN}$ Spearman's $r=0.18, P=0.57)$.
Changes of CXCR4 in MSCs after reaming nailing. The mRNA levels of CXCR4 receptor were compared between the two BM-MSC samples (BN and AN) obtained from the iliac crest. There was no significant change in the relative mRNA levels of CXCR4 receptor between the two samples $(0.06 \pm 2.1)$ (Fig. 3A). In detail, a mixed trend was observed with 2 out of 9 patients showing more than 3 -fold increase in CXCR4, 4 patients with moderate increase (less than 2-fold) and 3 patients with moderate decrease (less than 2-fold) in CXCR4 expression.

Furthermore, no statistically significant correlation was seen either between age or patient's gender or CXCR4 levels (Pearson's $\mathrm{r}=0.76, \mathrm{P}=0.4$ and Spearman's $\mathrm{r}=0.24, \mathrm{P}=0.53$, respectively). There was no correlation between the DT and CXCR4 mRNA expression (BN Spearman's $=-0.2, \mathrm{P}=0.58$, AN Spearman's $=-0.46, \mathrm{P}=0.15)$. Interestingly, comparison of the relative mRNA CXCR4 levels of MSCs obtained from the surgical site during RIN (IN) and the MSCs aspirated from the BM pool from the iliac crest $\mathrm{AN}$, a subset of patients showed that IN derived MSCs tended to have higher CXCR4 expression relative to the IC-BM derived cells following RIN (mean $\pm \mathrm{SD}$ : $\mathrm{IN}=0.24 \pm 0.35, \mathrm{AN}=-1.1 \pm 0.6$ ) (Fig. 3B).

Correlation of serum SDF-1 and MSC CXCR4 mRNA changes after reamed nailing. As shown above, SDF-1 increased in patient's sera and hence a possible correlation between SDF-1 in the serum and CXCR4 mRNA in MSCs was examined. An inverse correlation between SDF-1 in peripheral blood and CXCR4 in BM-MSCs following RIN was revealed. This observation suggested that the CXCR4 levels in MSCs are related to SDF-1 levels in the serum and possibly indicates the mobilization of MSCs through a decrease in the CXCR4/SDF-1 interaction in the IC-BM (Fig. 4).

\section{Discussion}

In a sudden event of a fracture, an inflammatory cascade is activated in order to achieve bone healing which starts from the formation of hematoma at the fractured site and ends in callus formation. RIN procedure represents a gold standard method for surgical treatment of diaphyseal long bone fractures for successful fracture stabilization and healing. This surgical treatment stabilization of the fractured bone requires the use of a device called intramedullary nail. The preparation of the bone canal is achieved with the aid of a sharp instrument. After the stabilization of the fracture site, a race starts for the callus formation. In addition, the RIN procedure, as a traumatic event 

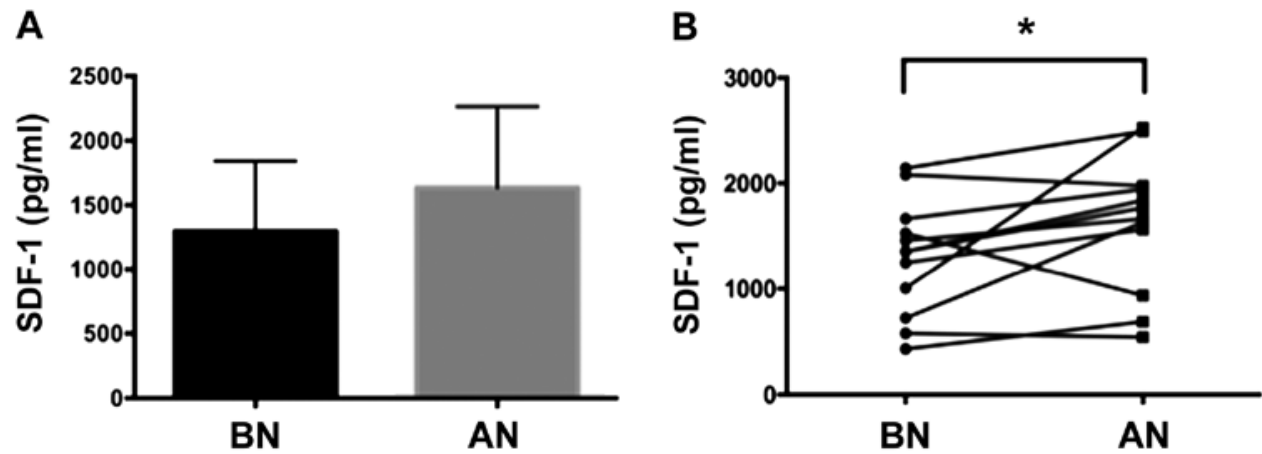

Figure 2. SDF-1 increases in the serum of patients following RIN. (A) Mean levels of SDF-1 measured by ELISA in sera of patients prior to and following RIN procedure. (B) Comparison of serum SDF-1 levels per patient. ("P<0.05, paired t-test). SDF-1, stromal cell-derived factor 1; RIN, reamed intramedullary nailing; BN, before the RIN; AN, after RIN.

A

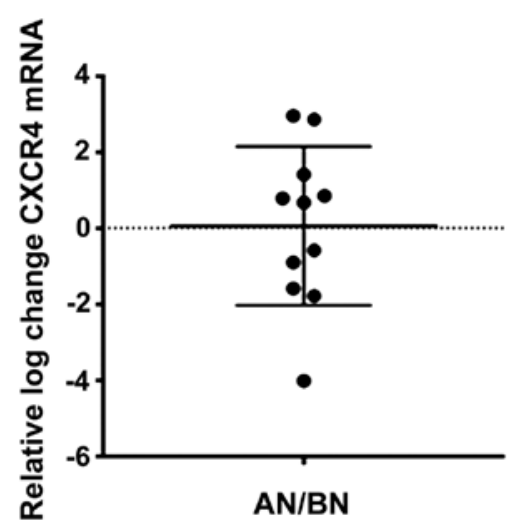

B

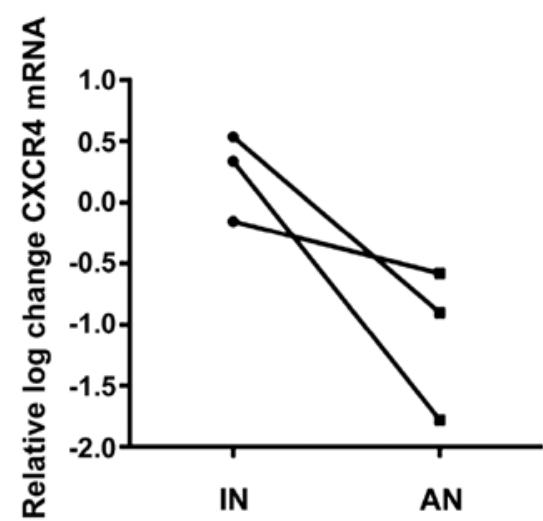

Figure 3. Change of CXCR4 mRNA following RIN. (A) CXCR4 mRNA levels in patients following RIN relative to baseline levels (BN). (B) CXCR4 mRNA levels between IN and AN samples relative to baseline levels (BN). CXCR4, C-X-C motif chemokine receptor 4; RIN, reamed intramedullary nailing; BN, before RIN; AN, after RIN; IN, intramedullary nailing.

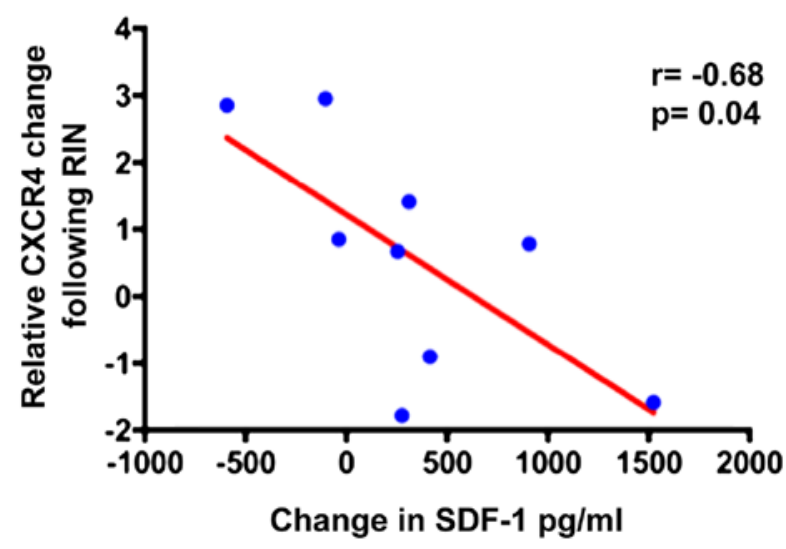

Figure 4. Inverse correlation of serum SDF-1 and MSC CXCR4 changes following RIN. Scatter plot of changes in CXCR4 mRNA levels relative to changes in SDF-1 serum protein levels (Pearson $r$ and P-value). SDF-1, stromal cell-derived factor 1 ; MSCs, mesenchymal stem cells; CXCR4, C-X-C motif chemokine receptor 4; RIN, reamed intramedullary nailing.

itself, participates in the inflammatory cascade. During RIN the so called debris is produced (6). The debris consists of MSCs which are able to promote bone healing. Their number increases in blood during organ and tissue injuries and other strenuous situations (21). MSCs are multipotent stromal cells capable of differentiating and contributing to the regeneration of mesenchymal tissues such as bone, cartilage, fat, tendon and muscle (22). MSCs are a source of progenitors for cell replacement and also activate or revive other local cells, such as tissue-resident progenitor or stem cells, endothelial cells and fibroblasts, to facilitate tissue regeneration via paracrine stimulation (23-26).

The movement of MSCs to injured tissues is described as endogenous stem cell homing and their subsequent participation to tissue repair is considered a natural self-healing response. In cases of injuries MSCs are recruited and mobilized into the damaged bone via local membrane and the peripheral circulation. There, MSCs enhance bone regeneration by differentiating directly into bone formation cells and modulating the biological environment by secreting growth factors and anti-inflammatory cytokines $(26,27)$. It has been demonstrated that MSCs migrate via the CXCR4 receptor to the fracture site and improve healing by affecting the biomechanical properties and increasing the cartilage and bone content of the callus (28). This migration is a multistep process which is mediated by homing receptors, endothelial co-receptors and chemotactic cytokines. The SDF-1/CXCR4 signaling axis has been demonstrated to be vital for MSCs homing (29).

The stress response after a fracture is the summary of physiologic response to the initial injury - the first hit, followed 
by the response to any ongoing surgical intervention such as RIN which represents the second hit (30). RIN provokes an inflammation as a cellular response that occurs during tissue injury due to the procedure. Inflammation is characterized by increased vascular permeability, recruitment of inflammatory cells, release of inflammatory mediators and turnover of matrices. That inflammatory wave is an important regulator for bone regeneration which initiates the repair cascade (31). Since MSC are implicated in tissue regeneration by recruitment to the inflammatory sites and the SDF-1/CXCR4 axis is vital for MSC homing and mobilization, this axis was selected to be investigated in order to examine the correlation, if any, to the RIN procedure and the contribution to fracture healing and callus formation $(32,33)$.

In order to examine the effect of RIN in the MSC BM pool, samples before and after the surgical procedure were aspirated from the iliac crest. In addition, blood samples were collected in parallel for the evaluation of the SDF-1 levels. The serum levels of SDF-1 were increased in peripheral blood following RIN and were associated with a decrease in CXCR4 mRNA in BM-MSCs. Taking into consideration that SDF-1 is a chemokine strongly chemoattractant to MSCs through its unique receptor CXCR4 (33) and has also been demonstrated highly expressed at injury sites and enhanced endochondral bone formation by homing MSCs (34,35), it could be suggested that RIN induces the rise of SDF-1 serum levels as long as the surgery represents a traumatic event for bone tissue at the injury site. Under normal circumstances MSCs and progenitor cells remain in the $\mathrm{BM}$ niche, where they are anchored due to the interaction between SDF-1 expressed in MSC niche and the CXCR4 receptor which is a membrane lipid (29). RIN as the second hit phenomenon promotes an inflammatory wave, provokes the release of several proteolytic enzymes such as MMPs and neutrophil elastase by granulocytes and monocytes and the enzymes affect the integrity of SDF-1/CXCR4 axis connection which acts as anchor for MSCs thus promoting their mobilization from BM niche (13). The present study showed that SDF-1 levels following RIN were increased which may be explained because SDF-1 is showed to be stimulated by hypoxic environment such as during RIN (36). The mobilization of BM stem cells by interfering with the axis SDF-1/CXCR4 interactions, has been demonstrated to enhance bone regeneration. SDF-1 secreted by BM-MSCs, bone endothelium, osteoblasts and stimulated proliferation and growth of B-cell progenitors represent the soluble factor for CXCR4 receptor at the surface of MSCs. This event at the initial stage of bone healing is crucial because the SDF-1 presence promotes osteoblast progenitors migration, differentiation and bone remodeling $(37,38)$.

Therefore, RIN through MSC migration under the action of CXCR4/SDF-1 axis may promote the fracture healing procedure through the gathering of multipotent MSCs to the 'damaged' and fractured bone site. The increased SDF-1 serum levels are reversely correlated to mRNA CXCR4 on BM-MSCs after nailing. This could possibly indicate mobilization of activated MSCs from the IC-BM pool. Activation of SDF-1 pathway could be expected due to inflammatory events that follow the surgical procedure and as a consequence of tissue damage. Our findings that SDF-1 levels increased after nailing point to this direction.
The mRNA levels of CXCR4 receptor after RIN, relative to the levels before the procedure (baseline) were also investigated. In contrast to the changes in SDF-1 serum levels, no specific trend in CXCR4 mRNA levels in MSCs following RIN was found and highly variable, patient-dependent response that included mixed trend of CXCR4 mRNA levels following RIN was observed (12). CXCR4 expression has been reported to be down-regulated in aged BM-MSCs, thus reducing their migration and anti-inflammatory capacity, however, we did not observe any correlation of patient's age or gender with CXCR4 changes (39). It could be hypothesized that the increase of SDF-1 expression following RIN, induces a cascade of mobilization through the decrease in expression of CXCR4 and subsequently reduction of MSC anchorage within the BM pool stimulating their migration. In support of this hypothesis we determined the CXCR4 mRNA expression of MSCs obtained directly from the site of the fractured limb. They conversely showed higher CXCR4 mRNA level expression compared to MSCs obtained from the iliac crest following RIN suggesting MSCs which possessed enhanced homing capacity (40).

The time interval between the incidence of the fracture and the RIN procedure ranged from 3 to $14 \mathrm{~h}$. This could be a limitation of our study since CXCR4 change was determined using BN sample as baseline and if there is a fluctuation in mRNA CXCR4 levels the different time windows among the patients may explain the mixed trend of their levels compared to baseline CXCR4. It could be hypothesized that CXCR4 mRNA changes may represent a different phase of cell migration under the effect of the axis CXCR4-SDF-1 that occurred prior to RIN. This is supported by previously reported data that the signals which regulate stem cells mobilization are often weakened or impaired because the function of SDF-1 is short lived (41).

There was no correlation between MSC count comparing the two time points, BN and AN. The absolute number of cells was not correlated with changes during the RIN. Also the dynamics of the cells for both samples (BN and AN), as represented by DT, did not show any statistically significant difference. RIN did not trigger MSC population to proliferate in a different way at these specific time points. There was no difference in fracture healing among the patients and the time to achieve callus formation was similar. Also, no nonunion or malunion was detected among the patients.

There are limitations in the present study. First, the adopted inclusion criteria and the type of bone fracture limited the number of included patients in the experimental study. Second, the femur or tibia fractures are not common or frequent diagnoses. However, this is a first attempt to investigate the connection of the axis CXCR4/SDF-1 with RIN, which based on the extracted results, should be expanded to a larger patient group. A future study could include the characterization of more MSC migration and homing pathways, which could further illuminate the molecular and biochemical effects of RIN in the MSC population.

In conclusion, the present study provides evidence of the effects of RIN on MSC population. The axis CXCR4/SDF-1 which contributes to MSC migration was selected and the levels of serum SDF-1 factor were shown to be elevated after the RIN. In addition, the increased levels of SDF-1 in peripheral blood were reversely correlated to the mRNA levels of CXCR4 on BM-MSCs after the RIN. It could be suggested that 
RIN induce an inflammation cascade through the phenomenon of second hit which increases the inflammation response within the BM niche. The enzymes digest the integrity of CXCR4/SDF-1 complex which is in balance and that event brakes the anchors of MSCs and facilitate the MSC migration. MSC migration happens through various expression of CXCR4 receptor at the surface of stem cells in response to the gradient of SDF-1 by chemoattaction. SDF-1 is the director of the attraction and migration of MSCs since it is released also from the injury site. The recruitment and homing of MSCs are essential for bone healing since MSC mobilization stimulates angiogenesis and bone remodeling.

\section{Acknowledgements}

Not applicable.

\section{Funding}

No funding was received.

\section{Availability of data and materials}

The datasets used and/or analyzed during the current study are available from the corresponding author on reasonable request.

\section{Authors' contributions}

IS, ET, CK, SM and THT collected the samples and carried out the experiments. IS and ET wrote the manuscript. DAS, KMA and GK contributed to the conception of the work and provided critical revision.

\section{Ethics approval and consent to participate}

Ethics committee of the University Hospital of Heraklion approved the protocol and all patients included in the study were informed and provided written consent.

\section{Patient consent for publication}

All patients provided written informed consent for publication of the results.

\section{Competing interests}

DAS is the Editor-in-Chief for the journal, but had no personal involvement in the reviewing process, or any influence in terms of adjudicating on the final decision, for this article. The other authors declare that they have no competing interests.

\section{References}

1. Zelle BA and Boni G: Safe surgical technique: Intramedullary nail fixation of tibial shaft fractures. Patient Saf Surg 9: 40, 2015

2. Hansen ST and Winquist RA: Closed intramedullary nailing of the femur. Küntscher technique with reaming. Clin Orthop Relat Res (138): 56-61, 1979.

3. Brookes M: Blood supply of long bones. BMJ 2: 1064-1065, 1963.

4. Cuthbertson EM, Siris E and Gilfillan RS: The femoral diaphyseal medullary venous system as a venous collateral channel in the dog. J Bone Joint Surg Am 47: 965-974, 1965.
5. Pape HC and Giannoudis P: The biological and physiological effects of intramedullary reaming. J Bone Joint Surg Br 89: 1421-1426, 2007.

6. Wenisch S, Trinkaus K, Hild A, Hose D, Herde K, Heiss C, Kilian O, Alt V and Schnettler R: Human reaming debris: A source of multipotent stem cells. Bone 36: 74-83, 2005.

7. Choumerianou DM, Dimitriou H and Kalmanti M: Stem cells: Promises versus limitations. Tissue Eng Part B Rev 14: 53-60, 2008.

8. Mansilla E, Marín GH, Drago H, Sturla F, Salas E, Gardiner C, Bossi S, Lamonega R, Guzmán A, Nuñez A, et al: Bloodstream cells phenotypically identical to human mesenchymal bone marrow stem cells circulate in large amounts under the influence of acute large skin damage: New evidence for their use in regenerative medicine. Transplant Proc 38: 967-969, 2006.

9. Shyu WC, Lee YJ, Liu DD, Lin SZ and Li H: Homing genes, cell therapy and stroke. Front Biosci 11: 899-907, 2006.

10. Ramírez M, Lucia A, Gómez-Gallego F, Esteve-Lanao J, Pérez-Martínez A, Foster C, Andreu AL, Martin MA, Madero L, Arenas J, et al: Mobilisation of mesenchymal cells into blood in response to skeletal muscle injury. Br J Sports Med 40: 719-722, 2006.

11. Kuznetsov SA, Mankani MH, Gronthos S, Satomura K, Bianco P and Robey PG: Circulating skeletal stem cells. J Cell Biol 153: 1133-1140, 2001.

12. Jones E and McGonagle D: Human bone marrow mesenchymal stem cells in vivo. Rheumatology (Oxford) 47: 126-131, 2008.

13. Ponte AL, Marais E, Gallay N, Langonné A, Delorme B, Hérault $\mathrm{O}$, Charbord $\mathrm{P}$ and Domenech $\mathrm{J}$ : The in vitro migration capacity of human bone marrow mesenchymal stem cells: Comparison of chemokine and growth factor chemotactic activities. Stem Cells 25: 1737-1745, 2007.

14. Ratajczak MZ and Kim C: Bioactive sphingolipids and complement cascade as new emerging regulators of stem cell mobilization and homing. J Stem Cell Res Ther 1: 1, 2011.

15. Ratajczak MZ: A novel view of the adult bone marrow stem cell hierarchy and stem cell trafficking. Leukemia 29: 776-782, 2015.

16. Tan HB, Giannoudis PV, Boxall SA, McGonagle D and Jones E: The systemic influence of platelet-derived growth factors on bone marrow mesenchymal stem cells in fracture patients. BMC Med 13: 6, 2015.

17. Antoniou KM, Papadaki HA, Soufla G, Kastrinaki MC, Damianaki A, Koutala H, Spandidos DA and Siafakas NM: Investigation of bone marrow mesenchymal stem cells (BM MSCs) involvement in Idiopathic Pulmonary Fibrosis (IPF). Respir Med 104: 1535-1542, 2010.

18. Kastrinaki M-C, Sidiropoulos P, Roche S, Ringe J, Lehmann S, Kritikos H, Vlahava VM, Delorme B, Eliopoulos GD, Jorgensen C, et al: Functional, molecular and proteomic characterisation of bone marrow mesenchymal stem cells in rheumatoid arthritis. Ann Rheum Dis 67: 741-749, 2008.

19. Karagiannis K, Proklou A,Tsitoura E,Lasithiotaki I,Kalpadaki C, Moraitaki D, Sperelakis I, Kontakis G, Antoniou KM and Tzanakis N: Impaired mRNA expression of the migration related chemokine receptor CXCR4 in mesenchymal stem cells of COPD patients. Int J Inflamm 2017: 6089425, 2017.

20. Livak KJ and Schmittgen TD: Analysis of relative gene expression data using real-time quantitative PCR and the $2^{-\Delta \Delta C T}$ method. Methods 25: 402-408, 2001.

21. Pountos I, Jones E, Tzioupis C, McGonagle D and Giannoudis PV: Growing bone and cartilage. The role of mesenchymal stem cells. J Bone Joint Surg Br 88: 421-426, 2006.

22. Jiang Y, Jahagirdar BN, Reinhardt RL, Schwartz RE, Keene CD, Ortiz-Gonzalez XR, Reyes M, Lenvik T, Lund T, Blackstad M, et al: Pluripotency of mesenchymal stem cells derived from adult marrow. Nature 418: 41-49, 2002.

23. Chen X, Armstrong MA and Li G: Mesenchymal stem cells in immunoregulation. Immunol Cell Biol 84: 413-421, 2006.

24. Ren G, Zhang L, Zhao X, Xu G, Zhang Y, Roberts AI, Zhao RC and Shi Y: Mesenchymal stem cell-mediated immunosuppression occurs via concerted action of chemokines and nitric oxide. Cell Stem Cell 2: 141-150, 2008.

25. Wang Y, Chen X, Cao W and Shi Y: Plasticity of mesenchymal stem cells in immunomodulation: Pathological and therapeutic implications. Nat Immunol 15: 1009-1016, 2014.

26. Zwingenberger S, Yao Z, Jacobi A, Vater C, Valladares RD, Li C, Nich C, Rao AJ, Christman JE, Antonios JK, et al: Enhancement of BMP-2 induced bone regeneration by SDF- $1 \alpha$ mediated stem cell recruitment. Tissue Eng Part A 20: 810-818, 2014. 
27. Gibon E, Yao Z, Rao AJ,Zwingenberger S, Batke B, Valladares R, Smith RL, Biswal S, Gambhir SS and Goodman SB: Effect of a CCR1 receptor antagonist on systemic trafficking of MSCs and polyethylene particle-associated bone loss. Biomaterials 33: $3632-3638,2012$

28. Granero-Moltó F, Weis JA, Miga MI, Landis B, Myers TJ, O'Rear L, Longobardi L, Jansen ED, Mortlock DP and Spagnoli A: Regenerative effects of transplanted mesenchymal stem cells in fracture healing. Stem Cells 27: 1887-1898, 2009.

29. Moll NM and Ransohoff RM: CXCL12 and CXCR4 in bone marrow physiology. Expert Rev Hematol 3: 315-322, 2010.

30. Giannoudis PV, Smith RM, Bellamy MC, Morrison JF, Dickson RA and Guillou PJ: Stimulation of the inflammatory system by reamed and unreamed nailing of femoral fractures. An analysis of the second hit. J Bone Joint Surg Br 81: 356-361, 1999.

31. Claes L, Recknagel S and Ignatius A: Fracture healing under healthy and inflammatory conditions. Nat Rev Rheumatol 8: 133-143, 2012.

32. Gerstenfeld LC, Cullinane DM, Barnes GL, Graves DT and Einhorn TA: Fracture healing as a post-natal developmental process: Molecular, spatial, and temporal aspects of its regulation. J Cell Biochem 88: 873-884, 2003.

33. Aiuti A, Webb IJ, Bleul C, Springer T and Gutierrez-Ramos JC: The chemokine SDF-1 is a chemoattractant for human CD34 hematopoietic progenitor cells and provides a new mechanism to explain the mobilization of $\mathrm{CD} 34^{+}$progenitors to peripheral blood. J Exp Med 185: 111-120, 1997.

34. Zlotnik A and Yoshie O: The chemokine superfamily revisited. Immunity 36: 705-716, 2012.
35. Yellowley C: CXCL12/CXCR4 signaling and other recruitment and homing pathways in fracture repair. Bone key Rep 2: 300, 2013.

36. Mohyeldin A, Garzón-Muvdi T and Quiñones-Hinojosa A: Oxygen in stem cell biology: A critical component of the stem cell niche. Cell Stem Cell 7: 150-161, 2010.

37. Xing Z, Lu C, Hu D, Yu YY, Wang X, Colnot C, Nakamura M, Wu Y, Miclau T and Marcucio RS: Multiple roles for CCR2 during fracture healing. Dis Model Mech 3: 451-458, 2010.

38. Shirley D, Marsh D, Jordan G, McQuaid S and Li G: Systemic recruitment of osteoblastic cells in fracture healing. J Orthop Res 23: 1013-1021, 2005.

39. Baker N, Boyette LB and Tuan RS: Characterization of bone marrow-derived mesenchymal stem cells in aging. Bone 70: 37-47, 2015.

40. Rombouts WJC and Ploemacher RE: Primary murine MSC show highly efficient homing to the bone marrow but lose homing ability following culture. Leukemia 17: 160-170, 2003.

41. Wei FY, Leung KS, Li G, Qin J, Chow SK, Huang S, Sun MH, Qin L and Cheung WH: Low intensity pulsed ultrasound enhanced mesenchymal stem cell recruitment through stromal derived factor-1 signaling in fracture healing. PLoS One 9: e106722, 2014.

(i) $\odot$ This work is licensed under a Creative Commons Attribution-NonCommercial-NoDerivatives 4.0 International (CC BY-NC-ND 4.0) License. 